\title{
Holographic Image Projection with Phase Only Spatial Light Modulators via Non- Iterative CGH Computation Method
}

\author{
Deniz Mengü, Erdem Ulusoy, Hakan Ürey \\ Koç University, Electrical Engineering Department and Optical Microsystems Laboratory, Istanbul, Turkey \\ *Corresponding author: erdemulusoy@ku.edu.tr
}

\begin{abstract}
A holographic image projection system that uses a non-iterative method for encoding computer generated holograms (CGH) on a phase-only spatial light modulator (SLM) is proposed. In each video frame, the method perfectly reconstructs a 2-to-1 sub-sampled version of the image pixels (such as even indexed rows), while a correlated encoding noise is distributed over the remaining pixels. By altering the sub-sampling grid among even and odd rows and columns between frames, the encoding noise is smoothly distributed over the image area. Experimental results suggest that the slightly degraded image quality is acceptable for the benefits of system simplicity and low computational cost.

OCIS codes: $090.1760,100.3010,070.0070,060.5060$.
\end{abstract}

\section{Introduction}

Holographic image projection (HIP) systems have been researched with growing interest due to their advantages over conventional image projection systems such as optical aberration correction, lens-less projection capability, increased light efficiency, etc. However, the absence of commercially available full-complex spatial light modulators (SLM) constitutes a challenge. A few full-complex SLM architectures have been proposed, but these are still at the research level [1,2].Therefore, procedures for encoding computer generated holograms (CGH) on currently available binary or phase-only SLMs are required. Common algorithms such as Iterative Fourier Transform Algorithm (IFTA), Gerschberg-Saxton, Fine-up, etc, are computationally expensive since multiple iterative steps are involved, requiring the usage of expensive ASICs or GPUs [3]. HIP systems based on high frame rate Ferroelectric Liquid Crystal (FLC) devices have been proposed where encoding noise is averaged over multiple sub-frames, each of which are computed using a single Discrete Fourier Transform (DFT) [4]. However, the solution has the disadvantage that it requires fabrication and precise alignment of certain phase masks with binary SLMs.

In this paper, we propose an HIP system that uses a non-iterative direct analytical method for encoding CGHs on a phase-only SLM. The method requires only a single DFT per each frame of a video sequence, removing the necessity of using expensive computational resources. At the same time, the generated noise is highly correlated with the image, and thus suitable for smoothing out with temporal averaging. Simulations and experiments verify that real-time performance is attainable via inexpensive computational resources with modest loss in quality.

\section{Proposed HIP System and CGH Computation Method}

In the HIP system we propose, we assume that the desired 2D image is obtained as the intensity distribution on the Fourier Transform plane of a phase-only SLM. If the SLM provided full complex modulation, given the desired image, $I[k, 1]$, we could determine the required SLM pattern as $S[m, n]=\mathrm{F}^{-1}\{\sqrt{I[k, l]}\}$ where ' $\mathrm{F}$ ' denotes Discrete Fourier Transform. Since this is not the case, we need to calculate $P[m, n]$ such that $|P[m, n]|=1$, and if $\mathrm{F}\{P[m, n]\}=A[k, l] e^{j \varphi[k, l]}, A^{2}[k, l]$ is as close as possible to $I[k, l]$. Note that the $e^{j \varphi[k, l]}$ term smooths the spectrum of the image so as to reduce the encoding error at the cost of speckle noise.

In our HIP system, we use a direct method for computation of phase-only CGH that relies on simple DFT relations related to multi-rate image processing. Fig.1 illustrates the basic principles of our method. Fig.1a shows an image consisting of letters $\mathrm{E}$ and $\mathrm{O}$, such that letter $\mathrm{E}$ is present on the even indexed rows and letter $\mathrm{O}$ is present on the odd indexed rows, as seen from the interlaced structure of the image. Fig.1b shows a full complex CGH that perfectly reconstructs the image in Fig.1a. Next, in Fig.1c, the even rows in Fig.1a (hence letter E) are preserved and the odd rows (hence letter $\mathrm{O}$ ) are eliminated. This sub-sampling, as well known, creates an overlapping effect in the 
dual domain. As shown, upper and lower parts of the CGH in Fig.1b are overlapped and added. This observation also implies that, if we form the CGH in Fig.1d from the CGH in Fig.1b, we sub-sample the image that the original CGH reproduces by perfectly preserving the even rows. Fig.1e and Fig.1f show the counterpart of Fig.1c and Fig.1d for odd rows.

a)

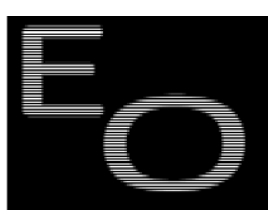

c)

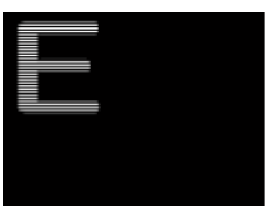

e)

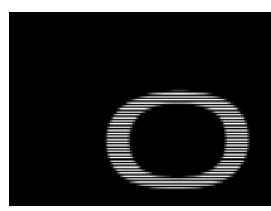

b)

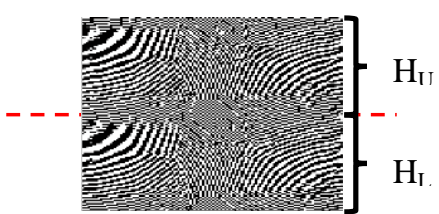

d)

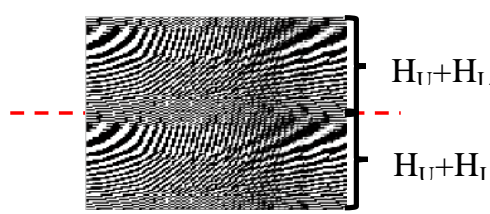

f)

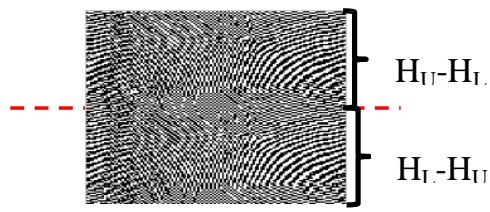

Figure 1: a) Fourier plane image. Letter ' $E$ ' is present on even indexed rows, while letter ' $O$ ' is present on odd indexed rows. b) Full-complex CGH that reconstructs (a). $\mathrm{H}_{U}$ and $\mathrm{H}_{\mathrm{L}}$ respectively denote upper and lower halves of the SLM. c) Even rows of (a). d) Full-complex CGH of (c). e) Odd rows of (a). f) Full-complex CGH of (e).

The observations in Fig.1 are used to compute phase-only CGHs, along with the fact that any complex number with a modulus between 0 and 2 can be obtained as the addition of two complex numbers of unit magnitude [5]. In particular, consider a phase-only hologram $\mathrm{P}_{\mathrm{E}}$ which satisfies $\mathrm{P}_{\mathrm{U}}+\mathrm{P}_{\mathrm{L}}=\mathrm{H}_{\mathrm{U}}+\mathrm{H}_{\mathrm{L}}$, as shown in Fig.2. Then, the observation in Fig.1c and Fig.1d indicates that $P_{E}$ perfectly reconstructs the even rows of the desired image.

However, since $\mathrm{P}_{U}-\mathrm{P}_{\mathrm{L}}$ is not necessarily equal to $\mathrm{H}_{U}-\mathrm{H}_{\mathrm{L}}, \mathrm{P}_{\mathrm{E}}$ generates a correlated noise image over the odd rows. In the end, $\mathrm{P}_{\mathrm{E}}$ becomes a phase-only $\mathrm{CGH}$ that reconstructs a sub-sampled version of the desired image perfectly, and corrupts the remaining part with a correlated noise. Similarly, a phase-only $\mathrm{CGH}, \mathrm{P}_{\mathrm{O}}$, can be computed that perfectly reconstructs the odd rows, as in Fig.2b. The methodology can be adapted in a straightforward manner to compute phase-only CGHs that perfectly reconstruct even or odd columns as well. Though a single CGH obtained in this manner reconstructs only part of the image in the desired way, when multiple such CGHs are displayed timesequentially, the noise will be smoothed over the image area, and the SNR and contrast ratios will improve.

\section{Experimental Results and Conclusion}

We conducted an experiment to verify our method. An 8-bit phase only SLM (Holoeye-Pluto) is illuminated by a converging spherical wave and a CCD array is placed on the Fourier plane over the area that lies between zeroth and first diffraction orders. Four phase-only CGHs are computed for a desired image using the method described in Section 2 for perfectly reconstructing even rows, odd rows, even columns, odd columns. The images generated by these CGHs are shown in Fig.3a-d. These images are averaged to obtain the image shown in Fig.3e, which has higher reconstruction quality and lower noise level compared to the individual images, indicating that when the CGHs computed for varying sub-sample grids are displayed consecutively, the encoding noise tends to smooth out. The greatest advantage of the proposed method is that it does not require any iterative steps, hence it is quite suitable for cost effective real time solutions. Moreover, the correlated nature of the encoding noise is advantageous in that spatial filter type noise removal solutions do not seem to be necessary. The method can be extended to other types of SLMs as well, such as binary SLMs, by changing the ratio of sub-sampling that is employed. This research has been supported by the European Research Council Advanced Grant ERC-2013-AdG- 111025 (WEAR3D). 
a)

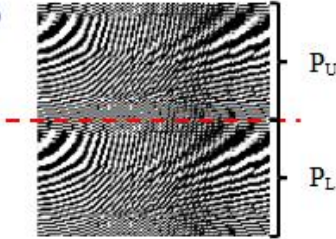

$P_{U}+P_{L}=H_{U}+H_{L}$

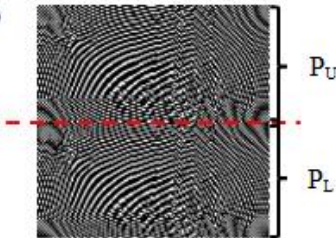

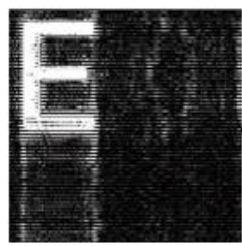

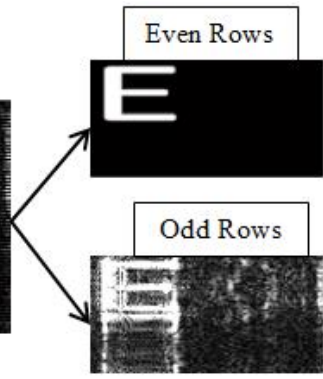

d)

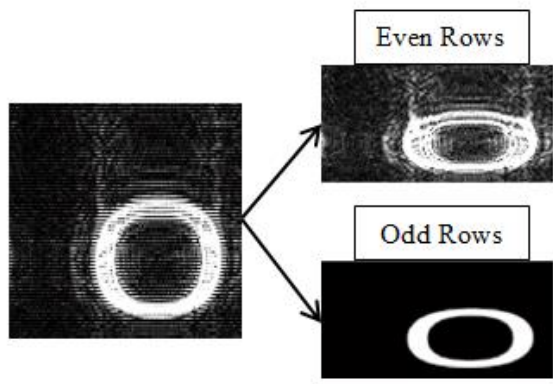

$P_{U}-P_{L}=H_{U}-H_{L}$

Figure 2: Phase hologram for exact reconstruction of even rows. b) Reconstructed image by (a). c) Phase hologram for exact reconstruction of odd lines. d) Reconstructed image by (c).
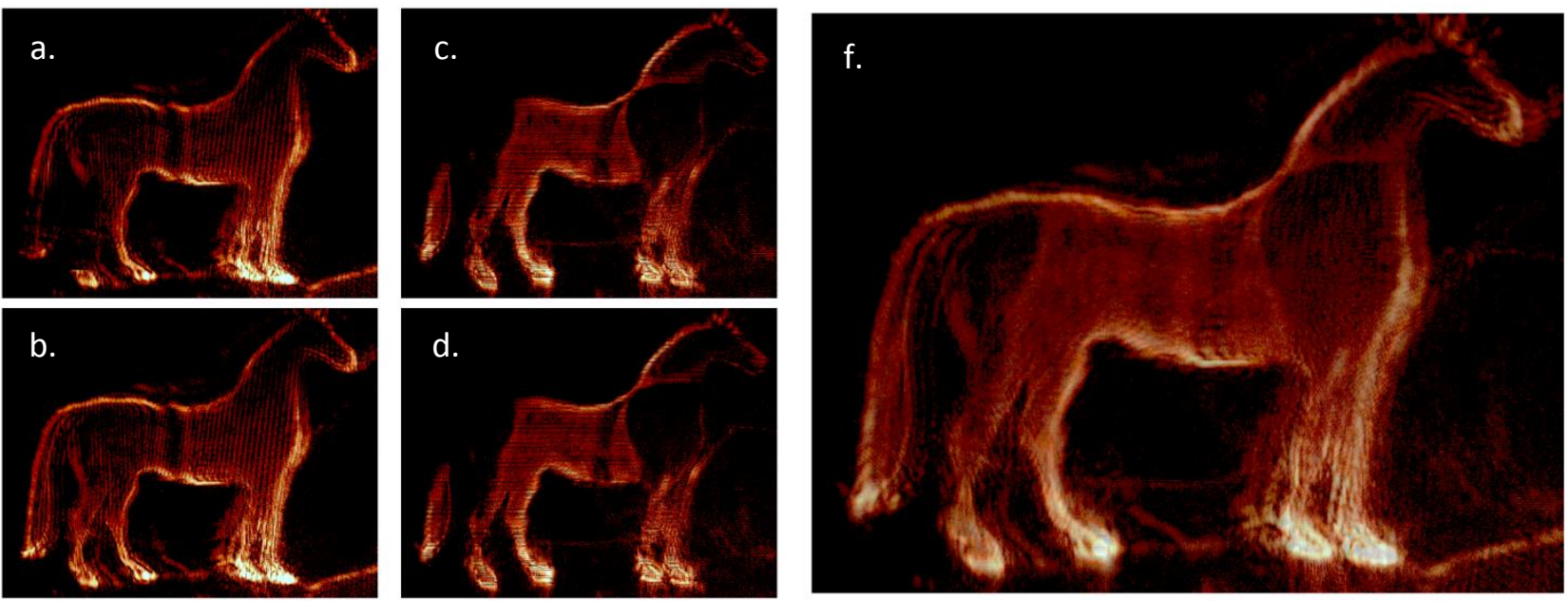

Figure 3: Image reconstructed by phase-only CGH that perfectly reconstructs a) even columns, b) odd columns, c) even rows, d) odd rows, e) average of (a),(b),(c),(d)

\section{References}

[1] Reichelt, Stephan, et al. "Full-range, complex spatial light modulator for real-time holography." Optics letters 37.11 (2012): $1955-1957$.

[2] Birch, Philip, et al. "Fully complex optical modulation with an analogue ferroelectric liquid crystal spatial light modulator." Optics communications 175.4 (2000): 347-352.

[3] Takada, Naoki, et al. "Fast high-resolution computer-generated hologram computation using multiple graphics processing unit cluster system." Applied optics 51.30 (2012): 7303-7307.

[4] Buckley, Edward. "70.2: Invited Paper: Holographic Laser Projection Technology." SID Symposium Digest of Technical Papers. Vol. 39. No. 1. Blackwell Publishing Ltd, 2008.

[5] Juday, Richard D., and James M. Florence. "Full-complex modulation with two one-parameter SLMs." San Diego,'91, San Diego, CA. International Society for Optics and Photonics, 1991. 